\title{
DYNAMIC THERMAL RATING FOR INCREASING NETWORK CAPACITY AND DELAYING NETWORK REINFORCEMENTS
}

\author{
Dave ROBERTS \\ ScottishPower Energy Networks - UK \\ dave.roberts@sppowersystems.com
}

\author{
Philip TAYLOR \\ Durham University - UK \\ p.c.taylor@dur.ac.uk
}

\author{
Andrea MICHIORRI \\ Durham University - UK \\ andrea.michiorri@dur.ac.uk
}

\begin{abstract}
Currently the operators of electrical distribution networks face a number of challenges, such as load growth, the proliferation of distributed generation and ageing infrastructure. This is drawing attention to techniques which will allow more efficient asset utilisation and carefully selected reinforcement projects.

This paper presents the findings of research into the technical challenges and potential benefits of dynamic thermal rating (DTR) systems. The adoption of DTR systems has the potential to improve network capacity and in some cases to offer an effective alternative to traditional reinforcement methods.

The research presented in this paper suggests that, when compared to traditional solutions of network reinforcement, a DTR system can, in some cases, offer a less invasive and more cost effective solution which allows the DNO to realise improved network component utilisation.
\end{abstract}

\section{INTRODUCTION}

Increasing energy demand and distributed generation (DG) coupled with stringent commercial and environmental considerations have caused DNOs to seek methods of increasing the utilisation of their existing assets. In the United Kingdom (UK) for example in order to meet the government's environmental targets for 2010, approximately $10 \mathrm{GW}$ of additional distributed generation will have to be connected to UK distribution networks [1]. This increased utilisation must be realised cautiously such that it does not reduce the security of supply to customers. This point is further emphasised when the age of distribution network assets is taken into account. In most western countries distribution networks were built between 1960 and 1980 in a period of economic growth associated with high energy consumption. It has been shown [2] that as the ageing process continues increased failure rates should be expected along with increased replacement costs.

Furthermore, difficulties are often encountered when attempting to gain permission to build new network infrastructure due to planning problems and environmental objections [3].

One potential solution or means of deferring these problems is the adoption of active management techniques which have the potential, in certain circumstances, to be both less invasive and more cost effective when compared to network reinforcement options. For example, the active management of generation or load can help to reduce power flow peaks preventing lines from reaching their voltage or thermal limits. Active network management techniques can also be used to increase the power flowing through a section of network such that its utilisation is increased in a safe manner. This increased utilisation can be realised within the normal static ratings but the focus of this work is to show how the adoption of dynamic thermal ratings could increase network utilisation further in a cost effective and safe manner.

\section{Related Work}

There are a number of research and development projects looking at the issue of dynamic thermal ratings throughout the world. An example of a DTR application in the transmission network in the region of Madrid is described in [4]. In this case, a low number of weather stations are used to estimate wind speed and direction over a wide geographical area; the real time rating of the overhead line (OHL), is then calculated.

The Electric Power Research Institute (EPRI) developed a similar system in the late 1990s considering OHLs, underground cables (UGCs) and power transformers (PTRs). In [5] and [6] the system and field test results are reported. It was found that for a complete network, rating increases of up to $15 \%$ of the static value were possible. OHLs, UGCs and PTRs are also examined in the DTR system described in [7]. Here component dynamic behaviour is taken into account and the advantages of DTRs for DNOs are described. Finally the advantages of DTR systems for accommodating distributed generation, in particular wind power, within the distribution network are described in [8] and [9] for OHLs and PTRs respectively. The work described in this paper differs from the work previously described in that it compares cost, transmission capacity and operational risk of a line with a DTR system and other reinforcement alternatives.

The paper is organized in the following way. Firstly a description of the DTR system under development at Durham University (DU) is given. This includes an overview of the thermal model used to rate OHLs. Then a case study network is presented and the results of the study are reported and discussed. Finally conclusions relating to the potential advantages of implementing a DTR system are drawn.

\section{DYNAMIC THERMAL RATING}

The dynamic thermal rating (DTR) concept is based on the observation that the first limit met for the current carrying capacity of a circuit is its temperature. This parameter is influenced by the ability of the component to dissipate to the environment the heat produced by the joule effect, and by external conditions such as ambient temperature, or wind speed. 
Due to the extreme variability and unpredictability of meteorological conditions, conservative conditions are currently used to determine the most appropriate component static rating. A DTR approach to network and load management consists of estimating or measuring component temperature or real current carrying capacity, in order to allow the power system component utilisation to be safely increased.

Research carried out at DU, aiming to develop active network management techniques involving the use of a DTR system, shows that there is an average exploitable headroom for OHLs, UGCs and PTRs in the region of $250 \%, 12 \%$ and $15 \%$ of the static component rating, respectively for typical UK climates. This is in agreement with the findings described in [5].

The DTR system under development at DU comprises a number of different weather stations and temperature measurement devices, placed in different locations over a wide network area. They are connected with a central computer responsible for calculating real time rating estimation. This information may then be used as a decision support tool for the secure operation of the distribution network. This computational device can be placed either in a remote substation or in a centralised control room.

\section{Component rating}

This paper focuses on the application of a DTR system for OHLs, but it has also been applied to UGCs and PTRs.

The fundamental concept behind the component rating is that the temperature limits of the power component must not be exceeded in order to avoid damaging the component. For OHLs in particular, a temperature rise leads to a reduction in conductor tension and to an increase in the sag. Typical values for maximum conductor temperature are between 50 ${ }^{\circ} \mathrm{C}$ and $90^{\circ} \mathrm{C}$.

Component temperature, is not a constant value but depends upon the energy balance between the heat produced inside the component and the heat exchange on its surface. The energy dissipated depends on the load, however the heat exchange is mainly influenced by the temperature difference between ambient and the environment and by other external factors such as wind speed or solar radiation.

Considering the heat dissipated by the Joule effect $\left(R I^{2}\right)$, the heat exchanged by convection $\left(Q_{c}\right)$ and radiation $\left(Q_{r}\right)$, and the solar radiation $\left(Q_{s}\right)$, the energy balance for an OHL conductor is described in Equation (1).

$$
R I^{2}+Q_{s}=Q_{c}+Q_{r},\left[W m^{-1}\right]
$$

The heat gained by solar radiation can be calculated as in Equation (2) considering solar radiation $\left(W_{s}\right)$, conductor diameter $(D)$ and an absorption coefficient $(\alpha)$

$$
Q_{s}=\alpha W_{s} D
$$

The radiative heat exchange depends on conductor temperature $\left(T_{c}\right)$, ambient temperature $\left(T_{a}\right)$, the StefanBoltzman constant $(\sigma)$ and an emission coefficient $(\varepsilon)$, as reported in Equation (3).

$$
Q_{r}=\varepsilon \sigma\left(T_{c}^{4}-T_{a}^{4}\right) \pi D
$$

Finally the convective heat exchange depends on air thermal conductivity $(\lambda)$ and the Nusselt number $(\mathrm{Nu})$

$$
Q_{c}=\pi N u \lambda\left(T_{c}-T_{a}\right)
$$

The Nusselt number can be calculated using wind direction correction factor $\left(K_{d i r}\right)$ and the Reynolds number $(R e)$ as in Equation (5).

$$
N u=K_{d i r} \cdot\left(0.65 \cdot \mathrm{Re}^{0.2}+0.23 \cdot \mathrm{Re}^{0.61}\right)
$$

The wind direction correction factor and the Reynolds number can in turn be calculated as in Equations (6) and (7) , using wind direction $\left(W_{d}\right)$, wind speed $\left(W_{s}\right)$ and empirical parameters $(A, B, C)$

$$
\begin{gathered}
K_{d i r}=A+B \sin ^{C}\left(W_{d}\right) \\
\operatorname{Re}=1.644 \cdot 10^{9} \cdot W_{s} \cdot D\left(\frac{T_{c}+T_{a}}{2}\right)^{-1.78}
\end{gathered}
$$

More information about the model described can be found in [11] and [12].

In Figure 1, the rating of a LYNX conductor exposed to different wind speeds is given. Using the model previously described, an air temperature of $20^{\circ} \mathrm{C}$, a conductor operating temperature of $50^{\circ} \mathrm{C}$ and a flow normal to the conductor axis are assumed.

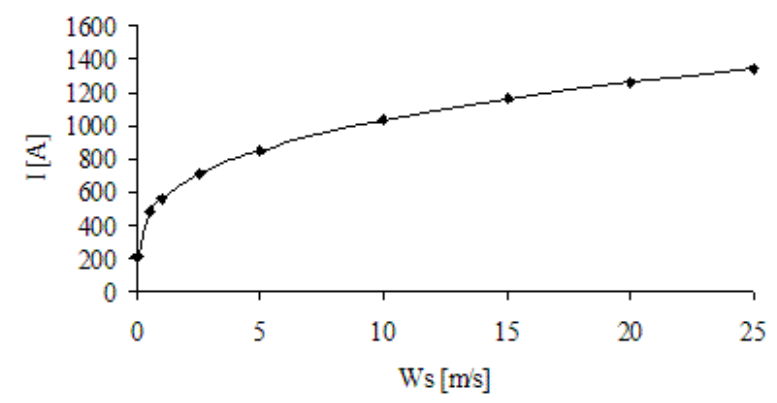

Figure 1: Rating variation of LYNX OHL with wind speed

\section{CASE STUDY}

\section{Network description: topology, conductors, load}

The case study network presented in Figure 2 was adapted from a portion of ScottishPower Energy Network (SPEN) network. A preliminary study was carried out for a $132 \mathrm{kV}$ Lynx conductor. The single line connects two towns that are $7 \mathrm{~km}$ apart, in an area attractive to prospective wind farm development. 


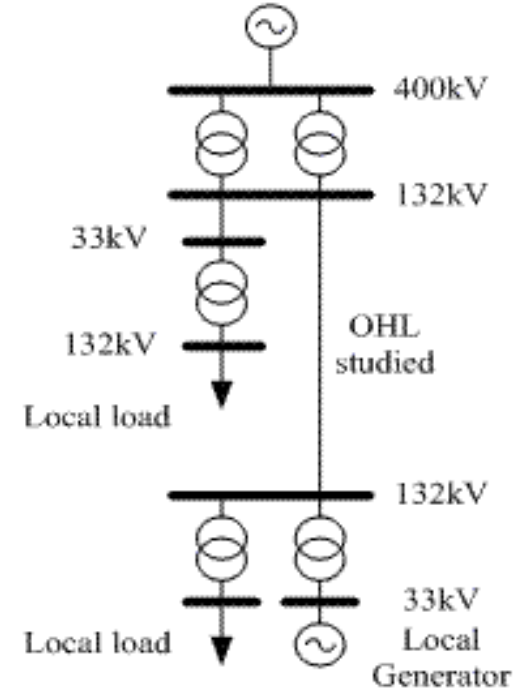

Figure 2: Case study network schematic diagram

This represents a typical situation where the possible installation of a wind farm or of an additional load is not influenced only by the wind resource or other environmental and legal issues, but also by the ability of the local network to absorb and/or export the energy produced.

\section{Methodology}

In the paragraph below preliminary results from research at DU are reported.

Hourly weather data from Valley (Wales, UK) have been used to estimate weather parameter values in each point of the line. These, in conjunction with the model described previously, have been used to calculate a series of real time thermal ratings for the studied line. The method reported in [11] has been employed for the OHL model, using the correction for wind direction reported in [12].

The real transmission capacity in MVA was then calculated and compared with the static rating currently in use, as well as other more conventional alternatives, such as retensioning the line or reinforcing the network.

Estimates of the costs of these alternatives are also given: re-tensioning costs are calculated from [15] and information from the planning department of SPEN was used to estimate new line construction costs. DTR system costs are estimated by the author.

In order to gain some understanding of the risk associated with the employment of a DTR system, hourly dynamic thermal rating simulation results where compared with hourly simulated load data. This was based on typical data at the site throughout 2005.

\section{$\underline{\text { Results and discussion }}$}

In Figure 3, the real time dynamic ratings are reported and compared with:

1) the static rating attributed to the same line,

2) the static rating of the line re-tensioned for operation up to $75^{\circ} \mathrm{C}$

3) the static rating of the new line for reinforcement with a "Upas" $300 \mathrm{~mm}^{2}$ AAAC conductor at an operational temperature of $75^{\circ} \mathrm{C}$

It is possible to see the great variability of real time rating across the year, the low value of the conservative static rating adopted compared to the real time rating and also the risk taken into account when the static rating is set. This underlines the value of a DTR system in preventing thermal overloads.

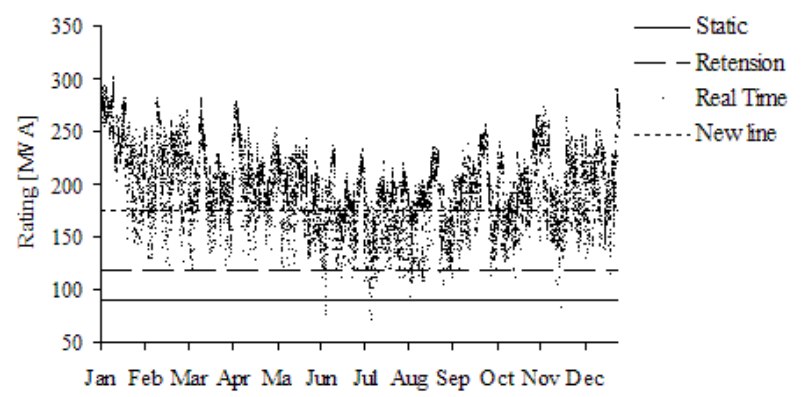

Figure 3: Real Time and static ratings comparison.

In Table 1 the results shown in Figure 3 are analyzed further, reporting the yearly energy transfer capacity in GWh for the different four different cases. This data is compared with the rating and the cost of each alternative solution. Ratings are taken from [13] and costs are based on the most appropriate data available at the time of consideration.

\begin{tabular}{llll}
\multicolumn{3}{c}{$\begin{array}{c}\text { Capability } \\
\text { Rating [A] [GWh/year] }\end{array}$} & Cost [M£] \\
\hline Static rating & 390 & 762 & 0 \\
Re-tensioned & 520 & 1034 & 0.16 \\
DTR & Variable & 1696 & 0.1 \\
New line & 770 & 1542 & 2
\end{tabular}

Table 1: Rating, maximum transmission capacity and cost.

In Table 2 the amount of time, in hours and the percentage of time when the real time rating is lower than the static rating, the rating of the re-tensioned line or the reinforced line rating is reported.

\begin{tabular}{lll} 
& {$[\mathbf{h}]$} & {$[\%]$} \\
\hline Real Time < Static & 7 & $0.08 \%$ \\
Real Time < Re-tensioning & 75 & $0.98 \%$ \\
Real Time < New line & 2855 & $32.59 \%$
\end{tabular}

Table 2: Time when real time rating is lower than static rating or alternative options.

The annual percentage time when the real time rating is lower than the static rating of the reinforced line may appear to be significant. However, the real time dynamic rating must be correlated with load variability to make an accurate assessment of the line utilization.

In Figure 4, the numbers of hours the load or the real time rating have a particular value are reported. By analysing these two parameters it is possible to assess the number of hours of potential overload.

By considering the probability of a particular circuit load coinciding with a rating smaller than the circuit load, it is possible to calculate the probability of incurring a thermal overload. In the example considered this event would take place for 56 hours per year. This is in agreement with further research at DU which identifies the maximum feasible uprating capacity of a Lynx conductor with a DTR 
system in the range of 150-180 MVA, according to climatic conditions. When this limit is passed, the number of thermal overload hours rises to unacceptable values.

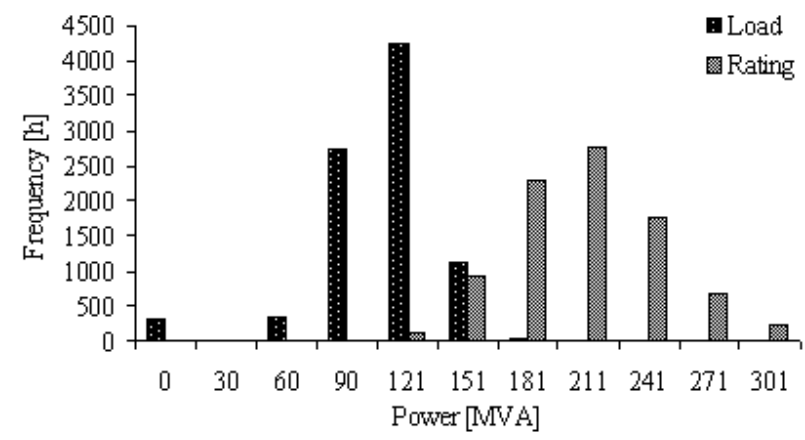

Figure 4: Load and rating frequencies over the year in hours

A more in depth analysis must take into account the accuracy of the DTR system. Ongoing research at DU aims to assess the precision of a DTR tool with theoretical and field measurement work. At the moment, without experimental results, it is not possible to provide an estimate for the precision of the DTR developed. This precision would depend in particular upon wind speed and wind direction estimation. Whilst errors in wind direction estimation would not affect the results for the whole year, imprecision in wind speed estimates could affect the findings of the work, by providing inaccurate line capability estimation.

\section{CONCLUSION}

Advantages and limitations arising from the implementation of a DTR system have been identified by comparing this approach to alternative re-tensioning or network reinforcement solutions. This serves to underline the potential benefits and drawbacks of a DTR approach.

From a comparison of installation costs and energy transfer capacity, a DTR system may be able to offer the greatest potential benefits with the lowest cost. In comparison with line re-tensioning, the adoption of a DTR system can provide a $67 \%$ gain in energy transfer capacity at $62 \%$ of the re-tensioning cost.

Moreover, by calculating the hours of potential thermal overload, a DTR system may allow a line to transfer power up to twice that the value of its static rating.

It can be concluded that the use of a DTR system has the potential to assist with the accommodation of larger power flows in the existing infrastructure, whilst at the same time increasing operational security.

\section{ACKNOWLEDGMENTS}

The authors wish to acknowledge the Department for Innovation, Universities and Skills for funding, and the staff from ScottishPower, AREVA T\&D, Imass, Parsons Brinckerhoff and the Met Office for the help provided.

\section{REFERENCES}

[1] Mott MacDonald, 2003, "Capacity mapping and market scenarios for 2010 and 2020"

[2] Y.C. Wijnia, M.S. Korn, S.Y. de Jager, P.M. Herder, 2006, "Long term optimization of asset replacement in energy infrastructures" Systems, Man and Cybernetics, IEEE International Conference on, vol.3, 2615-2621

[3] P. Fox-Penner, 2001, "Easing Gridlock on the Grid - Electricity Planning and Siting Compacts", The Electricity Journal, vol. 14 (9), 11-30

[4] F. Soto, J. Latorre, M. Wagensberg, 1998, "Increasing the capacity of OHLs in the $400 \mathrm{kV}$ Spanish transmission network: real time thermal ratings", Electra vol. 22-211

[5] D.A. Douglass, A.A. Edris, 1996, "Real-time monitoring and dynamic thermal rating of power transmission circuits", IEEE Transactions on Power Delivery, vol. 11 (3), 1407-1418

[6] D.A. Douglass, A.A. Edris, 1997, "Field application of a dynamic thermal circuit rating method", IEEE Transactions on Power Delivery, vol. 12 (2), 823-831 [7] J.M.A. Nuijten, A. Geschiere, 2005, "Future network planning and grid control" International Conference on Future Power Systems, Amsterdam

[8] P.D. Belben, C.D. Ziesler, 2002, "Aeolian uprating: how wind farms can solve their own transmission problems", World Wind Energy Conference and Exhibition, Berlin

[9] M. Helmer, 2000, "Optimized size of wind power plant transformer and parallel operation", Wind Power for the 21 st century, Kassel, Germany

[10] A. Neumann, P. Taylor, S. Jupe, A. Michiorri, A Goode, D. Curry, D. Roberts, 2008, "Dynamic thermal rating and active control for improved distribution network utilisation", Powergrid Europe Conference and Exhibition, Milan

[11] IEC, 1995, “IEC/TR 61597 - Overhead electrical conductors - calculation methods for stranded bare conductors"

[12] WG 22.12, 1992, "The Thermal Behaviour of Overhead Conductors - Section 1". Electra, vol. 144-3

[13] Scottish Power Transmission and Distribution, 2005, Distribution Long Term Development Statement for SP Manweb plc for the years 2005/06 to 2009/10" 